\title{
Modelling coronal electron density and temperature profiles of the Active Region NOAA 11855
}

\author{
J. M. Rodríguez Gómez ${ }^{1}$, L. E. Antunes Vieira ${ }^{1}$, A. Dal Lago ${ }^{1}$, \\ J. Palacios ${ }^{2}$, L. A. Balmaceda ${ }^{3}$ and T. Stekel ${ }^{1}$ \\ ${ }^{1}$ National Institute for Space Research (INPE), \\ Avenida dos Astronautas-12227-010, São José dos Campos-SP, Brazil \\ email: jenny.gomez@inpe.br \\ ${ }^{2}$ Departamento de Física y Matemáticas, Universidad de Alcalá University Campus, Sciences \\ Building, P.O. 28871, Alcalá de Henares, Spain \\ ${ }^{3}$ Instituto de Ciencias Astronómicas de la Tierra y el Espacio, ICATE-CONICET, Avda. de \\ España Sur 1512, J5402DSP, San Juan, Argentina.
}

\begin{abstract}
The magnetic flux emergence can help understand the physical mechanism responsible for solar atmospheric phenomena. Emerging magnetic flux is frequently related to eruptive events, because when emerging they can reconnected with the ambient field and release magnetic energy. We will use a physic-based model to reconstruct the evolution of the solar emission based on the configuration of the photospheric magnetic field. The structure of the coronal magnetic field is estimated by employing force-free extrapolation NLFFF based on vector magnetic field products (SHARPS) observed by HMI instrument aboard SDO spacecraft from Sept. 29 (2013) to Oct. 07 (2013). The coronal plasma temperature and density are described and the emission is estimated using the CHIANTI atomic database 8.0. The performance of the our model is compared to the integrated emission from the AIA instrument aboard SDO spacecraft in the specific wavelengths $171 \AA$ and $304 \AA$.
\end{abstract}

Keywords. Sun: abundances, atmosphere, magnetic field.

\section{Introduction}

Flux emergence phenomena play a key role in the dynamics of the solar atmosphere. The physical processes involved in flux emergence contribute to a better understanding of magnetic evolution, transport processes, solar dynamo and emission over the solar cycle. Also, the problem of active region heating requires precise measurements of plasma parameters such as density and temperature (Tripathi et al. 2008), but direct measurements are difficult to acquire. Modelling these parameters from photospheric magnetic field are necessary. It is important to understand the relationship of coronal structures, magnetic field, and emission in specific wavelength and their relationship with the Earth's atmosphere. Here we show some preliminary ideas because this is a work is in progress.

\section{Data}

We have used data from SDO/HMI SHARPs (Bobra et al. 2014 and Hoeksema et al. 2014), from Sept. 29 (2013) to Oct. 07 (2013) related to the AR NOAA 11855. Images were corrected and aligned with standard procedures through SolarSoftWare (SSW). We used a data cube every six hours, ie. at 05:59 UT, 11:59 UT, 17:59 UT and 23:59 UT. We used the AIA images for some specific wavelengths $171 \AA$ and $304 \AA$ during 

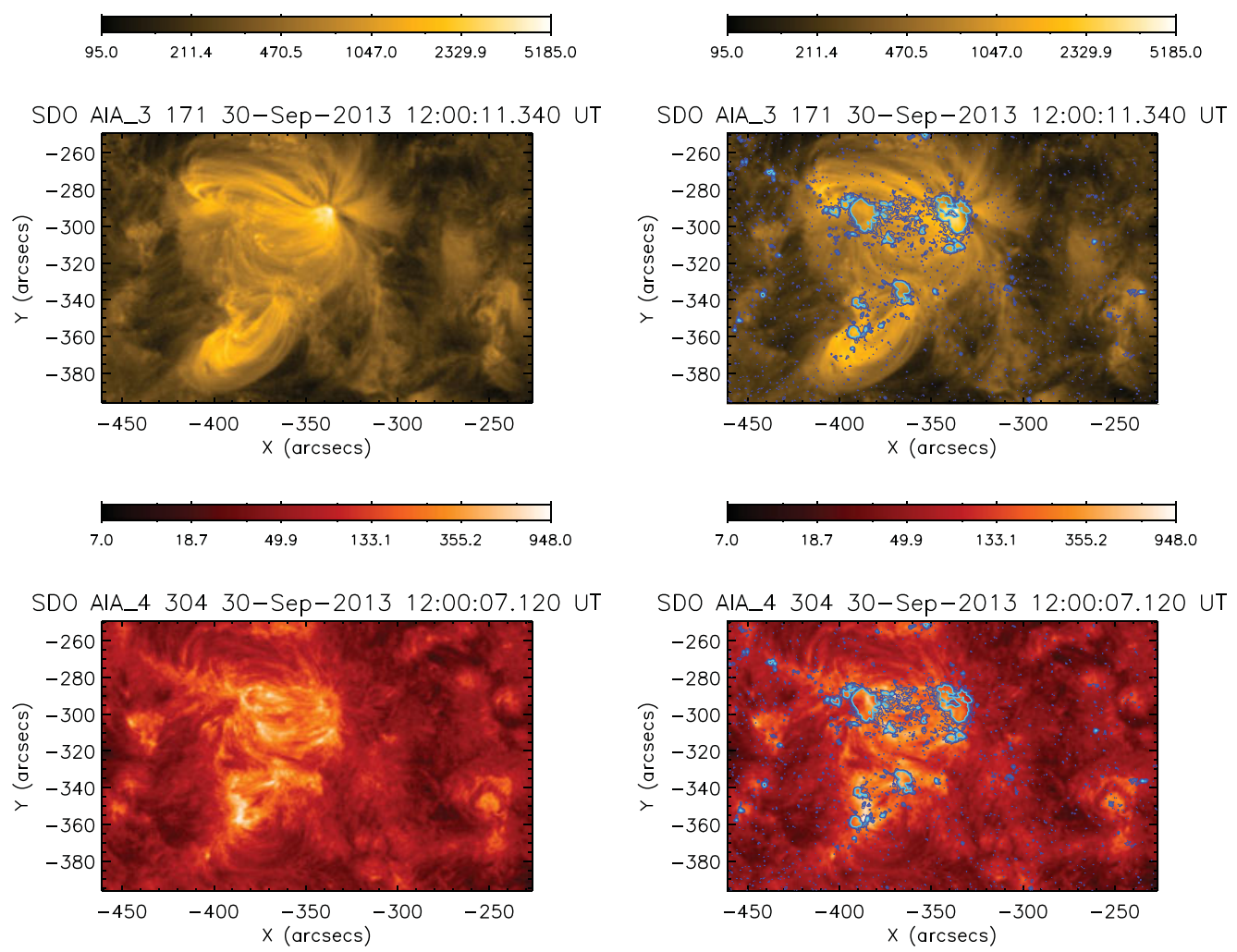

Figure 1. Active Region NOAA 11855. Upper panel: Images at $171 \AA$ from AIA/SDO and composite images using photospheric magnetic field from HMI/SDO for the same region Sept. 30 12:00:00 UT. Lower panel: Images at 304A from AIA/SDO and composite images using photospheric magnetic field from HMI/SDO for the same region Sept. 30 12:00:00 UT.

the period of interest. The region is selected and extracted. The AIA/SDO images are then corrected and aligned with standard procedures through SolarSoftWare (SSW) (left panels in Fig. 1). We used SHARPS magnetic field data overimposed to AIA (right panels in Fig. 1).

\section{Photospheric and coronal dynamics}

Non-linear force-free-field extrapolation of the solar magnetic field is used through the optimization method available by NLFFF in SSW (Wheatland et al. 2000). The magnetic field through the lower corona was obtained from $1 R_{\odot}$ to $1.15 R_{\odot}$. This magnetic field is an important key to modelling plasma parameters through the solar corona. The electron density $(N)$ and temperature $(T)$ profiles are described using the following expressions: $N=N_{o}(B)^{\gamma}$ and $T=T_{o}(B)^{b-1} e^{-\left(\frac{B}{a}\right)^{(b-1)}}$. Temperature profile is based on the Weibull distribution function, where $a, b$ and $\gamma$ are proportional coefficients, $N_{o}$ and $T_{o}$ are background density and temperature. From the images of AIA/SDO (left panel in Fig. 1) it is possible to calculate the integrated intensity in different heights of the solar atmosphere. We will use an emission model based on the contribution function and integrated line-of-sight emission. This emission is compared to the emission from AIA/SDO. This procedure allows modelling the electron density and temperature in the emergence flux region until the AR is developed. This diagnosis can be characterised by 

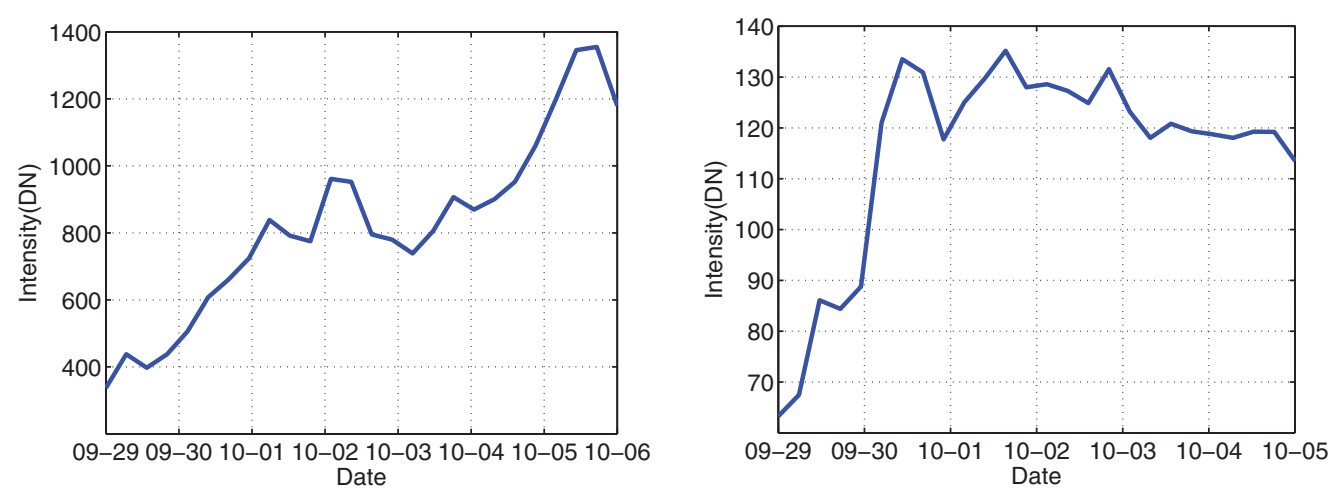

Figure 2. Integrated emission of NOAA 11855 from the images of the AIA instrument on board SDO spacecraft. Left panel: integrated intensity at 171 A AIA/SDO from Sept. 29 18:00 UT to Oct.06 18 : 00 UT. Right panel: Integrated intensity at 304Å AIA/SDO from $09-2918$ : 00UT to $10-0518: 00 \mathrm{UT}$.

these plasma parameters and evaluate the relationship with the evolution of photospheric features (flux emergence and ARs).

\section{Discussion}

Using these specific wavelengths we will obtain a description of the emission in the solar corona related to the active region NOAA 11855. These wavelengths describe the emission from the hot loops in $171 \AA$ and from the active region in the lower transition region in $304 \AA$. The emission is not linked to flares. The intensity increase is steeper in $304 \AA$ that in $171 \AA$ as we can see from the faster flux emergence emergence in the upper chromosphere, as compared to the corona (Fig. 2). In the next steps, we will include the extrapolated magnetic field in our model. We will obtain the density and temperature profiles and discuss the behavior of the emission in the lower solar corona related to the emergence flux region and Active Regions (ARs).

\section{Acknowledgements}

This work is partially supported by $\mathrm{CNPq} /$ Brazil under the grant agreement no. 140779/2015-9 and no. 304209/2014-7. JP acknowledges UAH travel grant for visiting INPE and MINECO project no. AYA2013-47735-P.

\section{References}

Bobra, M. G., Sun, X., Hoeksema, J. T., Turmon, M., Liu, Y., Hayashi, K., Barnes, G. \& Leka, K. D. 2014, Solar Phys., 289, 9

Hoeksema, J. T., Liu, Y., Hayashi, K., Sun, X., Schou, J., Couvidat, S., Norton, A., Bobra, M., Centeno, R., Leka, K. D., Barnes, G., \& Turmon, M. 2014, Solar Phys., 289, 3483

Tripathi, D., Mason, H. E., Young, P. R., \& Del Zanna, G. 2008, A\& A, 481, 1

Wheatland, M. S., Sturrock, P. A., \& Roumeliotis, G. 2000, ApJ, 540, 2 